Furthermore, the results of this study provide some evidence that no one measure of quality should be used itself to represent different aspects of the quality of primary care. Some health authorities are now producing performance indicators for the general practices they administer. ${ }^{8}$ Therefore, these performance measures must be interpreted appropriately. Further large studies need to be carried out to establish the impact of personal continuity on clinical care of patients with asthma.

\section{References}

1. Freeman G K and Richards F C. How much personal care in full group practices?. BMJ 1990; 301: 1028-30.

2. Freeman G. Priority given by doctors to continuity of care. $J R$ Coll
Gen Pract 1985; 5: 423-6.

3. Hjortdahl $\mathrm{P}$ and Laerun E. Continuity of care in general practice: effect on patients satisfaction. BMJ 1992; 304: 1287-90.

4. Sweeney K G and Gray D P. Patients who do not receive continuity of care from their general practitioner - are they a vunerable group? $\mathrm{Br} J$ Gen Pract 1995; 45: 133-5.

5. Breslau N and Reeb K G. Continuity of care in the university-based practice. J Med Aduc 1975; 50: 965-9.

6. Lakhani M, Baker R, Khunti K. Monitoring asthma. Eli Lilly

National Clinical Audit Centre. Leicester, University of Leicester, 1994.

7. Feder G, Griffiths C, Highton C et al. Do clinical guidelines

introduced with practice education improve care of asthmatic and

diabetic patients? A randomised controlled trial in general practices in east London. BMJ 1995; 311: 1473-8.

8. Majeed F A and Voss S. Performance indicators for general practice. BMJ 1995; 311: 209-10.

\title{
Myopia in an atopic patient
}

\author{
A Sheikh
}

\section{CASE REPORT}

A 21 year old student presented to his GP in 1991 with a three year history of increasing blurring of vision. During this time he had been repeatedly assessed by his optician who had diagnosed myopia and astigmatism. Glasses and contact lenses of increasing strengths had been prescribed with little benefit. Following the last visit the optician had written to his GP advising referral to an ophthalmologist for further assessment. A review of his history revealed that he was atopic suffering from asthma, eczema, allergic conjunctivitis and perennial rhinitis. There was no other history of note and no family history of eye disorders.

Assessment at the local eye hospital confirmed the profound myopia with a visual acuity of $6 / 36$ and $6 / 24$ in the right and left respectively. Keratoscopy revealed the findings of corneal irregularity and thinning characteristic of keratoconus. Rigid gaspermeable contact lenses were prescribed and a corrected visual acuity of $6 / 9$ and $6 / 6$ was achieved.

\section{Discussion}

Keratoconus was first described in $1854 .{ }^{1}$ It is a relatively common degenerative disease of the cornea with a prevalence of approximately 1 in $2000 .^{2}$ The condition affects all races with a slight preponderance for females. The pathological defect lies in Bowman's layer of the cornea; typical findings include keratocyte degeneration and disruption.

In approximately $6-8 \%$ of cases there is a positive family history; both autosomal dominant and recessive patterns of inheritance have been identified. ${ }^{3}$ For the others, no specific cause has yet been identified. Associations have been noted with a number of systemic and ocular disorders including Down's syndrome, Marfan's syndrome and retinitis pigmentosa. The most frequently described association, however, is with atopic conditions such as asthma, eczema and hay fever.
The evidence in support of an association between keratoconus and atopy is reasonably strong. Since the initial reported association in the early $1930 \mathrm{~s},{ }^{4} \mathrm{a}$ number of case reports have appeared in the literature describing the coexistence of atopic conditions and keratoconus..$^{5-7}$ This data has been further supported by descriptive studies that have consistently shown a high prevalence of atopic conditions in keratoconus patients. In a US study of 162 patients with keratoconus an $18 \%$ prevalence of asthma, and $35 \%$ prevalence of hay fever was observed. ${ }^{8}$ A British study of 67 patients revealed a history of atopic disease in $56 \%$ of patients; $28 \%$ of this group had asthma. ${ }^{9}$ More recently, in the largest study of its kind involving 38 centres and 1,579 patients, the Collaborative Longitudinal Evaluation of Keratoconus group reported atopic disease in $34.6 \%$ of patients, with $13 \%$ diagnosed as suffering from asthma. ${ }^{10} \mathrm{In}$ the above cases, the prevalence of asthma and atopy was significantly higher than that of the general population.

Data from controlled studies is less uniform. Lowel et al. in their small study of 31 cases failed to detect any significant difference in atopic traits between keratoconus patients and a similar number of unmatched controls. ${ }^{11}$ Their failure to detect a significant difference may have been due to a lack of power of the study. In a larger study of 182 cases and 100 matched controls, atopy was observed in $35 \%$ compared with $12 \%$ of the controls. The most common allergic disease encountered was hay fever, followed by asthma and eczema. It was also noted that serum $\operatorname{IgE}$ was significantly raised $(\mathrm{p}<0.001)$ in the keratoconus group and markedly so in those cases with associated atopic disease. ${ }^{12}$

Whilst the exact nature of the association between atopy and keratoconus is unclear, the frequency of their co-existence suggest that this is not simply a chance observation. Excessive eye rubbing by atopic patients has been suggested as a possible cause although the evidence in support of this is very



Asthma in Gen Pract 1998; 6(1): 13-14 
limited. ${ }^{13}$ More controlled studies are needed to clarify further the relationship between these conditions. Better designed studies are also required to ensure that atopic status has been adequately confirmed through detailed allergy history and appropriate skin prick testing; most of the studies thus far conducted have relied on history alone.

Keratoconus characteristically presents in the second decade of life with a gradual decline in visual acuity. It has a tendency to progress for about seven or eight years and then remain stable, but this varies considerably. The condition is almost invariably bilateral, though frequently more advanced on one side than the other. Clinical signs include a cone shaped cornea, indentation of the lower lid by the cornea when the patient looks down (Monson's sign) and an irregular reflex on retinoscopy. Diagnosis is usually confirmed by keratoscopy which remains the most sensitive diagnostic tool. ${ }^{14}$

Keratoconus is eminently treatable in the majority of cases. In the early stages of the disease adequate visual correction can often be achieved using ordinary glasses. With more advanced cases hard contact lenses are frequently required. Corneal grafting may be needed if a patient is unable to tolerate contact lenses or if the visual correction the lenses provide is inadequate. If a corneal transplant is performed before extreme corneal thinning the prognosis is excellent with about $80-95 \%$ obtaining reading vision.
I suggest that keratoconus be considered in all atopic patients presenting with progressive myopia

\section{References}

1. Nottingham G. Practical Observations on Conical Cornea. London, 1854

2. Krachmer J H, Feder R S, Belin M W. Keratoconus and related non-inflammatory corneal thinning disorders. Surv Ophthalmol 1984; 28: 293-322.

3. Redmond K B. The role of heredity in keratoconus. Trans Ophthalmol Soc NZ. 1968; 27: 52.

4. Hilgartner H D, Hilgartner H L, Gilbert J T. Keratoconus successfully treated with organotherapy, radium and short-wave diathermy. Am J Ophthalmol 1937; 20: 1032.

5. Brunsting L A, Reed M B, Blair H L. Occurrence of cataracts and keratoconus with atopic dermatitis. Arch Dermatol 1955; 72: 347.

6. Galin M and Berger R. Atopy and keratoconus. Am J Ophthalmol 1958; 45: 904.

7. Spencer W H and Fisher J J. The association of keratoconus with atopic dermatitis. Am J Ophthalmol 1959; 47: 332.

8. Gasset A R, Hinson W A, Frias J L. Keratoconus and atopic disease. Ann Ophthalmol 1978; 19(8): 991-4.

9. Harrison R J, Klrard P T, Fasty D L et al. Association between keratoconus and atopy. Br J Ophthalmol 1989; 73(10): 816-22. 10. Zadnik K, Barr J T, Gordon M O et al. Biomicrocopic signs and disease severity in keratoconus. Cornea 1996; 15: 139-46. 11. Lowell F C and Carroll J M. A study of the occurence of atopic traits in patients with keratoconus. J Allergy Clin Immunol 1970; 46: 32-9.

12. Rahi A, Davies $P$, Ruben $M$ et al. Keratoconus and coexisting atopic disease. Br J Ophthalmol 1977; 61: 761-4.

13. Gritz D C and McDonald P J. Keratoconus and ocular massage. Am J Ophthalmol 1988; 106: 757-8.

14. Maguire L J and Bourne W D. Corneal topography of early keratoconus. Am J Ophthalmol 1989; 108: 107-12.

\section{Shared care for asthma}

Mark Levy, John Couriel, Roland Clark, Stephen Holgate and Anoop Chauhan. Oxford, Isis Medical Media, 1997.

This book is aimed at busy healthcare professionals involved in the care of asthma patients, many of whom would be attracted by the title. It is, on the whole, easy-to-read with many useful figures and diagrams and some very apt cartoons.

The importance of good communication between the professionals involved, particularly at the primary-secondary care interface, is emphasised. Quite properly the authors stress the importance of continuity of care and proper training for all involved. There are two excellent chapters full of useful tips, examples of successful shared care protocols and well chosen case histories.

It is a shame that there is only a very brief mention of the role of school nurses and health visitors and none of others like community pharmacists and patient participation groups.

More than half of the book is about diagnosis and management and there is a particularly turgid chapter on the molecular mechanisms of asthma. How relevant is this to the people likely to read it?

I enjoyed reading this book, which as an introduction to asthma care is excellent, but has it got the right title? Less than half is truly about what I understand by shared care.

John Stanger

General Practitioner, Cambridgeshire
This book aims to bridge the gap between primary and secondary care for asthma patients. It is well structured and enhanced by clear diagrams and amusing illustrations throughout. A co-ordinated approach to asthma care can be achieved by capitalising on the individual skills of the health care professionals involved and improving communications between them.

Definition and epidemiology of asthma is followed by clear, easy to digest review of current views on its pathophysiology. A comprehensive chapter on diagnosis is welcomed with diagnostic difficulties constituting a high proportion of patients referred to hospital outpatients. Good guidance on steps to make the diagnosis, benefits of early diagnosis and prompt treatment are presented. The section on management includes avoidance of precipitants, classes of drugs used and devised as in line with national guidelines, but also introduces new approaches, eg. leukotriene antagonists.

Presented are examples of good and bad practice in communications across the interface eg. the referral letter, hospital discharge letter etc. together with case histories in an effective way to illustrate these points

All health professionals working in asthma care will find much of relevance and use in this book. Adoption of its examples of good practice will clearly work towards the provision of a seamless service of good quality for our asthmatic patients. 